\title{
Frontières
}

\section{Dans quelles conditions est-il acceptable ou non de mourir par euthanasie?}

\section{Isabelle Marcoux}

Volume 20, numéro 1, automne 2007

La « bonne mort »

URI : https://id.erudit.org/iderudit/017950ar

DOI : https://doi.org/10.7202/017950ar

Aller au sommaire du numéro

\section{Éditeur(s)}

Université du Québec à Montréal

ISSN

1180-3479 (imprimé)

1916-0976 (numérique)

Découvrir la revue

Citer cet article

Marcoux, I. (2007). Dans quelles conditions est-il acceptable ou non de mourir par euthanasie ? Frontières, 20(1), 69-75. https://doi.org/10.7202/017950ar
Résumé de l'article

L'opinion publique est de plus en plus favorable à la pratique de l'euthanasie dans un contexte de maladie incurable et de souffrances, ce qui constitue un argument prisé par les défenseurs de l'euthanasie. Cette étude démontre que la population québécoise y est majoritairement favorable, mais qu'une proportion importante émet des réserves dans d'autres contextes comme lorsque la personne ne souffre pas, lorsqu'il y a espoir de guérison, lorsque le motif de la demande est la souffrance psychologique ou morale, ou lorsque la personne est seulement très âgée. Selon les conditions de vie et de santé proposées, des caractéristiques sociodémographiques différentes semblent liées au fait de changer ou non d'opinion. D'autres études doivent être menées afin de mieux comprendre ce qui caractérise l'opinion de la population québécoise sur l'euthanasie et d'en suivre l'évolution. 


\section{Résumé}

L'opinion publique est de plus en plus favorable à la pratique de l'euthanasie dans un contexte de maladie incurable et de souffrances, ce qui constitue un argument prisé par les défenseurs de l'euthanasie. Cette étude démontre que la population québécoise y est majoritairement favorable, mais qu'une proportion importante émet des réserves dans d'autres contextes comme lorsque la personne ne souffre pas, lorsqu'il y a espoir de guérison, lorsque le motif de la demande est la souffrance psychologique ou morale, ou lorsque la personne est seulement très âgée. Selon les conditions de vie et de santé proposées, des caractéristiques sociodémographiques différentes semblent liées au fait de changer ou non d'opinion. D'autres études doivent être menées afin de mieux comprendre ce qui caractérise I'opinion de la population québécoise sur l'euthanasie et d'en suivre l'évolution.

Mots clés: euthanasie opinion publique - espoir de guérison souffrance psychologique personnes âgées.

\begin{abstract}
Growing public support towards the practice of euthanasia in situations of painful terminal illnesses provides a strong argument widely used by proponents for legalized euthanasia. This study reveals that the majority of Quebec's population would be in favor of euthanasia for incurable and suffering patients, but showed limited support in other cases such as when the patient is not suffering, when there is hope for recovery, when the main reason is psychological suffering or when a person has simply reached an old age. Different socialdemographic characteristics appear associated with changes in opinion depending on the proposed case scenarios. Other studies must be conducted to observe the evolution of public opinion toward euthanasia in Quebec in order to reach a better understanding of this complex issue.
\end{abstract}

Keywords: euthanasia - public opinion - hope of recovery psychological suffering - elders.

\section{Dans quelles conditions est-il acceptable ou non de mourir par euthanasie?}

\section{Isabelle Marcoux ${ }^{1}$, \\ Équipe de recherches en sciences humaines et sociales appliquées à la cancérologie, Université de Nantes, Centre de recherche sur le suicide et l'euthanasie, Université du Québec à Montréal.}

Bien que le terme euthanasie signifie «mort douce» dans son sens étymologique premier, il renvoie plutôt dans les milieux législatif, médical et de la recherche à l'acte de provoquer intentionnellement la mort d'une personne malade, à sa demande, pour mettre fin à ses souffrances; cet acte étant en principe accompli par un médecin. Depuis quelques décennies, l'opinion publique est de plus en plus favorable à une telle pratique et cet état de fait est un argument prisé par les défenseurs de la légalisation de l'euthanasie. Les études antérieures suggèrent toutefois que de telles attitudes sont complexes et changeantes et qu'elles méritent d'être analysées rigoureusement afin d'alimenter un débat plus éclairé sur cette question.

$\mathrm{Au}$ Canada, les opinions sur l'euthanasie se sont passablement modifiées au cours des dernières décennies avec un appui de la population en faveur de sa légalisation qui est passé de $45 \%$ en 1968 à $79 \%$ en 2002 (Gallup Canada, 1968-). Ce changement dans l'opinion publique vers un appui majoritaire a également été observé dans d'autres pays tels que l'Angleterre (O'Neill et al., 2002), l'Australie (Roy Morgan Research Center, 1996), les États-Unis (DeCesare, 2000) et les Pays-Bas (van der Maas et al., 1995). Plusieurs facteurs historiques et sociaux sont évoqués pour expliquer une telle transformation des mentalités dont l'avancement de la technologie médicale, le délaissement des croyances religieuses, l'adoption de valeurs morales plus individualistes, le vieillissement de la population, etc. (Mullens, 1994). Malgré cet appui majoritaire grandissant, certains auteurs ont émis des critiques quant à la validité des résultats des sondages: les gens seraient confus sur ce qu'est réellement l'euthanasie (Silveira et al., 2000; Emanuel et al., 1998; McInerney et al., 1995) et des erreurs méthodologiques dans la formulation des questions inciteraient certaines personnes à répondre de manière plus positive (Marcoux et al., 2007). Toutefois, il a été démontré que même lorsque ces sources potentielles d'invalidation sont contrôlées, les gens demeurent majoritairement favorables à l'euthanasie (Marcoux et al., 2007).

Au-delà de ces variations associées à des biais méthodologiques, les gens auraient des opinions diverses selon certaines conditions spécifiques. Tout d'abord, l'opinion varierait selon l'identité de la personne impliquée avec un appui comparable que la décision soit prise pour soi ou pour une personne fictive (Achille et al., 1997), mais un appui moindre lorsqu'il s'agit d'une personne proche comparativement à soi (Williams et al., 2007; Wade et al., 1987). L’identité de la personne qui assiste jouerait également un rôle puisque la population semble plus favorable à 
l'euthanasie ou à l'aide au suicide lorsque l'assistance est prodiguée par un médecin que lorsqu'il s'agit d'un proche (O'Neill et al., 2003 ; MacDonald, 1998). Certaines études se sont également intéressées à certaines caractéristiques de la personne malade et ont démontré que le pronostic du patient (Singer et al., 1995), son âge (enfant/adulte) ainsi que le type de maladie dont il est atteint (cancer / Alzheimer) (MacDonald, 1998) seraient des facteurs importants dans le fait de considérer l'euthanasie comme étant acceptable ou inacceptable. Dans des situations de dépression chronique ou de personnes âgées qui se sentent un fardeau pour les proches, l'appui à l'euthanasie serait beaucoup moins favorable que dans une situation de phase terminale avec des douleurs sévères (Genuis et al., 1994). Eu égard à l'évolution des mentalités sur la question de l'euthanasie, il est toutefois possible que de telles opinions se soient modifiées avec le temps puisque certaines de ces études ont été menées il y a plus de dix ans.

Des informations quant aux conditions de vie et de santé à l'égard desquelles l'euthanasie est considérée acceptable ou non sont importantes puisqu'elles mettent en perspective les résultats de sondage sur cette question et peuvent également nous permettre de suivre l'évolution des mentalités quant à ces conditions à travers le temps. Aux Pays-Bas, où l'euthanasie est permise sous certaines conditions depuis 1990 et légalisée depuis 2000, les critères d'admissibilité ont été modifiés pendant cette période pour y inclure entre autres la notion de souffrance psychologique. Cette situation avait créé une vive controverse auprès du public et même parmi les partisans de l'euthanasie (Sénat du Canada, 1995), bien qu'aujourd'hui elle soit acceptée légalement parmi les critères de minutie. À cet effet, certaines personnes sont réfractaires à la légalisation de l'euthanasie puisque, selon elles, une porte ouverte à sa pratique aurait pour conséquence une acceptation croissante des raisons et situations pour lesquelles l'euthanasie est acceptable (argument de la «pente glissante»). Aucune donnée ne permet toutefois d'infirmer ou de corroborer une telle hypothèse.

Dans le cadre de cette étude, un premier objectif consistait à vérifier jusqu'à quel point l'opinion de la population québécoise sur l'euthanasie est stable en examinant, parmi ceux qui considèrent l'euthanasie comme acceptable, les conditions dans lesquelles cette opinion peut varier. Nous avons tout d'abord étudié l'opinion selon deux situations où les conditions de base généralement associées à l'euthanasie sont absentes, c'est-à-dire lorsqu'il n'y a pas de souffrance et lorsqu'il y a espoir de gué- rison. Deux conditions pour lesquelles l'opinion sur l'euthanasie est plus partagée ont également été investiguées, à savoir l'euthanasie sur la base du critère de l'âge et lorsque la personne souffre psychologiquement. Comme deuxième objectif, nous avons ensuite vérifié si certaines caractéristiques sociodémographiques sont liées à un changement d'opinion selon ces conditions de vie et de santé.

\section{MÉTHODE}

\section{DEVIS ET RÉPONDANTS}

La collecte de données a été réalisée par une firme de sondage privée auprès d'un échantillon représentatif de la population québécoise dans le cadre d'une enquête omnibus portant sur différents sujets sociopolitiques. Les répondants devaient être âgés de 18 ans et plus et être aptes à répondre aux questions en français ou en anglais. Une stratification de l'échantillon par région (Montréal métropolitain $=50 \%$; Québec métropolitain $=20 \%$; reste du Québec $=30 \%$ ) a été réalisée afin de mieux représenter l'hétérogénéité des populations urbaines. La sélection des répondants a comporté deux étapes. Tout d'abord, les ménages ont été échantillonnés grâce à la méthode de génération aléatoire de numéros de téléphone qui permet de rejoindre les numéros répertoriés dans l'annuaire téléphonique et les numéros non répertoriés (par ex. : numéros récemment attribués et numéros confidentiels) afin d'obtenir un échantillon plus représentatif de la population. La deuxième étape consistait à sélectionner le répondant à l'intérieur du ménage. Lorsqu'un ménage comprend plus d'une personne, il faut s'assurer que ces personnes ont une chance égale d'être choisies. Pour ce faire, une grille de sélection basée sur le critère de l'âge (appelée grille Kish) a été utilisée (Lavrakas, 1987). Des entretiens téléphoniques ont été réalisés auprès de 991 répondants ce qui correspond à un taux de réponse de 49,8\% pour l'ensemble de la province (Montréal métropolitain =47,8\%; Québec métropolitain $=48,2 \%$; reste du Québec $=55,2 \%$ ) et ce, en tenant compte du taux d'admissibilité qui consiste en la proportion des unités admissibles (incluant le nombre d'entrevues complétées, les refus de la personne, les incomplets, les absences prolongées) par rapport aux unités valides (comprenant les unités admissibles précédemment énumérées plus les unités valides mais hors échantillon). Pour cette taille d'échantillon, avec un seuil de confiance de $95 \%$, étant donné l'estimation maximale de 50\% de la présence du comportement dans la population, la marge d'erreur est estimée à 3,1 points.

\section{MESURES}

Afin de mesurer l'acceptabilité de l'euthanasie selon diverses conditions, les répondants devaient tout d'abord se prononcer sur l'acceptabilité ou non de l'euthanasie en répondant à notre question générale: «Une personne atteinte d'une maladie incurable demande à son médecin de mettre fin à ses souffrances et de causer sa mort en lui donnant une injection de médicaments. Trouvez-vous qu'il est tout à fait acceptable, plutôt acceptable, plutôt inacceptable ou tout à fait inacceptable qu'un médecin donne une injection qui cause la mort à la demande de la personne? » Cette question prend en compte certains des indicateurs qui sont généralement considérés comme inhérents à la définition de l'euthanasie et qui sont utilisés dans la plupart des sondages sur l'euthanasie, à savoir: 1) l'intention est de provoquer la mort; 2) le motif est celui de mettre fin aux souffrances; 3) le geste qui cause la mort est posé par une personne autre que celle qui décède; et 4) la mort survient dans le contexte d'une maladie incurable. Des distinctions ont ensuite été proposées aux personnes qui ont répondu de manière affirmative à la question de l'acceptabilité générale de l'euthanasie ${ }^{2}$. Ceux-ci devaient indiquer s'ils considèrent comme acceptable ou non l'euthanasie dans un contexte où : $a$ ) la personne est atteinte d'une maladie incurable mais qu'elle ne souffre pas; $b$ ) il y a espoir de guérison; $c$ ) la personne n'est pas atteinte d'une maladie physique mais elle a des souffrances psychologiques ou morales continuelles; $d$ ) la personne est très âgée. À noter que cette étude aborde la notion de l'euthanasie dans un contexte médical seulement, c'est-à-dire telle qu'elle est pratiquée par un médecin, et ce, tout en tenant compte de la volonté de la personne telle qu'elle est prise en compte par la demande de cette dernière.

Des renseignements sur les caractéristiques sociodémographiques, culturelles et religieuses ont également été recueillis afin de mieux comprendre ce qui peut influencer certaines personnes à changer d'opinion selon ces diverses conditions de vie et de santé. Les caractéristiques sociodémographiques sont le genre, l'âge, le niveau de scolarité complété ( 7 années ou moins [primaire]; 8 à 12 années [secondaire]; 13 à 15 années [cégep/collège, école technique]; 16 années et plus [université]) et le revenu (moins de $20000 \$ ; 20000$ à 39999 ; $40000 \$$ à $59999 \$ ; 60000 \$$ à $79999 \$$; $80000 \$$ et plus). Le lieu de résidence (Île de Montréal/autres régions) et la langue maternelle (français / anglais / autre) constituent les variables dites culturelles dans le contexte québécois et sont généralement corrélées à la religion qui a été investiguée par l'identification à une religion (catholique 
/ autre religion/aucune religion) et la fréquence de la pratique religieuse (une fois par semaine ou plus; environ une fois par mois; quelques fois par année; une fois par année ou moins; jamais).

\section{ANALYSES STATISTIQUES}

Afin de s'assurer que les répondants à l'étude soient représentatifs de la population adulte du Québec venant des ménages privés ${ }^{3}$, les résultats ont été pondérés selon le lieu de résidence afin de compenser pour la stratification, et ont été ajustés selon l'âge, le genre et la langue d'usage à la maison sur la base de la Projection 2002 de Statistique Canada. La pondération consiste à attribuer à chaque répondant un poids relatif qui correspond au nombre de personnes qu'il représente dans la population. La comparaison des données pondérées et non pondérées sur ces caractéristiques a révélé que les répondants sont proportionnellement représentatifs de la population québécoise ${ }^{4}$.

La variable dépendante "l'opinion selon diverses conditions» a été codifiée, pour chacune des quatre situations présentées (pas de souffrance, espoir de guérison, souffrance psychologique ou morale, personne très âgée), en trois catégories afin de comparer ceux qui changent d'opinion selon ces conditions avec ceux qui ne changent pas d'opinion. Ces trois catégories sont: 1) ceux qui considèrent toujours l'euthanasie inacceptable;2) ceux qui changent d'opinion selon la condition; 3) ceux qui considèrent toujours l'euthanasie acceptable, peu importe la condition. Les personnes qui ont refusé de répondre à la question ou qui ne savaient pas quoi répondre ont été exclues. Ces quatre variables d'opinion ont donc été croisées avec chacune des variables sociodémographiques, culturelles et religieuses; le test de chi carré a été utilisé dans le cas de variables nominales et l'analyse de variance pour les variables continues. Afin de déterminer quelles caractéristiques liées à l'individu permettent d'expliquer un changement d'opinion tout en contrôlant pour l'effet des autres variables, une analyse de régression multinomiale a été effectuée pour chacune des questions d'opinion. La méthode backward stepwise a été utilisée afin d'obtenir la prédiction maximale eu égard aux variables étudiées ${ }^{5}$.

\section{RÉSULTATS}

Dans une situation où une personne atteinte d'une maladie incurable demande à son médecin de soulager ses souffrances en causant sa mort par une injection de médicaments, 69,6\% des personnes sondées jugent la pratique de l'euthanasie acceptable (dont $40,3 \%$ tout à fait acceptable et 27,6\% plutôt

TABLEAU 1

L'OPINION SUR L'EUTHANASIE SELON DIVERSES CONDITIONS (N=692)

\begin{tabular}{|c|c|c|c|}
\hline & \multicolumn{2}{|c|}{$\begin{array}{c}\text { Données } \\
\text { non pondérées }\end{array}$} & \multirow{2}{*}{$\begin{array}{c}\text { Données } \\
\text { pondérées } \\
\%\end{array}$} \\
\hline & $n$ & $\%$ & \\
\hline \multicolumn{4}{|c|}{ Lorsque la personne ne souffre pas } \\
\hline $\begin{array}{l}\text { Oui } \\
\text { Non } \\
\text { Ça dépend } \\
\text { NSP / Refus }\end{array}$ & $\begin{array}{r}263 \\
379 \\
37 \\
13\end{array}$ & $\begin{array}{r}38,0 \\
54,8 \\
5,3 \\
1,9\end{array}$ & $\begin{array}{r}37,6 \\
54,9 \\
5,9 \\
1,6\end{array}$ \\
\hline \multicolumn{4}{|c|}{ Lorsqu'il y a espoir de guérison } \\
\hline $\begin{array}{l}\text { Oui } \\
\text { Non } \\
\text { Ça dépend } \\
\text { NSP / Refus }\end{array}$ & $\begin{array}{r}162 \\
493 \\
32 \\
5\end{array}$ & $\begin{array}{r}23,4 \\
71,2 \\
4,6 \\
0,7\end{array}$ & $\begin{array}{r}22,6 \\
71,6 \\
5,0 \\
0,8\end{array}$ \\
\hline \multicolumn{4}{|c|}{ Lors de souffrances psychologiques ou morales continuelles } \\
\hline $\begin{array}{l}\text { Oui } \\
\text { Non } \\
\text { Ça dépend } \\
\text { NSP / Refus }\end{array}$ & $\begin{array}{r}193 \\
444 \\
29 \\
26\end{array}$ & $\begin{array}{r}27,9 \\
64,2 \\
4,2 \\
3,8\end{array}$ & $\begin{array}{r}29,8 \\
62,4 \\
4,4 \\
3,4\end{array}$ \\
\hline \multicolumn{4}{|c|}{ Lorsque la personne est très âgée } \\
\hline $\begin{array}{l}\text { Oui } \\
\text { Non } \\
\text { Ça dépend } \\
\text { NSP / Refus }\end{array}$ & $\begin{array}{r}218 \\
443 \\
19 \\
12\end{array}$ & $\begin{array}{r}31,5 \\
64,0 \\
2,7 \\
1,7\end{array}$ & $\begin{array}{r}31,5 \\
63,7 \\
3,0 \\
1,8\end{array}$ \\
\hline
\end{tabular}

acceptable), 25,1\% la considèrent comme inacceptable (dont $11,5 \%$ plutôt inacceptable et $13,6 \%$ tout à fait inacceptable), tandis que $5,2 \%$ ont soit refusé de répondre ou n'avaient pas d'opinion. Ceux qui ont jugé cette pratique acceptable devaient ensuite préciser s'ils conservent ou changent leur opinion selon différentes conditions de vie et de santé (voir tableau 1). Parmi ces 692 répondants, c'est lorsque la personne est atteinte d'une maladie incurable mais qu'elle ne souffre pas qu'une plus grande proportion d'entre eux maintiennent leur opinion (37,6\%). Si l'on considère l'échantillon total, environ $27 \%$ des gens sont en faveur de l'euthanasie si la personne ne souffre pas. Dans une situation où la personne est très âgée mais qu'elle n'est pas atteinte d'une maladie incurable, $31,5 \%$ maintiennent leur opinion si c'est la personne elle-même qui le demande, ce qui représente environ $22 \%$ de l'ensemble des répondants. Dans une proportion similaire $(29,8 \%)$, les gens demeurent favorables à l'euthanasie si la personne n'est pas atteinte d'une maladie physique mais qu'elle a des souffrances psychologiques ou morales continuelles, ce qui représente un appui de près de 20\% de l'ensemble des répondants de l'étude. Enfin, c'est lorsqu'il y a espoir de guérison $(22,6 \%)$ que les gens changent le plus d'opinion, $16 \%$ de l'échantillon total considèrent l'euthanasie comme acceptable dans une telle situation.
De manière générale, les résultats des régressions multinomiales sont semblables à ceux obtenus en analyses bivariées, et ils permettent avec une meilleure précision de statuer sur la qualité et l'ampleur des différences significatives entre le groupe d'intérêt (ceux qui changent d'opinion selon les conditions) et ceux qui conservent leur opinion (toujours acceptable ou toujours inacceptable). Pour ces raisons, et par souci de concision, seuls les résultats des régressions multinomiales sont présentés dans les tableaux 2 à 5 ; les résultats des analyses bivariées ne seront présentés et discutés que lorsqu'ils diffèrent des résultats des analyses multivariées.

Avec la méthode backward stepwise, seules les variables significatives à $p<0,05$ sont retenues dans le modèle. Ainsi, pour les quatre questions d'opinion, le genre, le niveau de scolarité complété et le lieu de résidence ont été exclus. Dans la situation où une personne demande l'euthanasie mais qu'elle ne souffre pas (tableau 2), le modèle retenu explique $15 \%$ de la variance et contient les variables suivantes: l'âge, le revenu, la langue maternelle, l'identification et la pratique religieuse. Ainsi, comparativement aux personnes qui ont changé d'opinion, celles qui considèrent toujours l'euthanasie comme inacceptable ont un revenu moins élevé (1,2 fois), elles sont plus nombreuses à s'identifier à une religion autre que catholique [référence $=$ athée] 
TABLEAU 2

RÉGRESSION MULTINOMIALE DE L'OPINION SUR L'EUTHANASIE LORSQUE LA PERSONNE NE SOUFFRE PAS ( $\mathrm{N}=927)$

\begin{tabular}{lcccc}
\hline $\begin{array}{l}\text { Groupe de référence: } \\
\text { Change d'opinion }\end{array}$ & \multicolumn{2}{c}{$\begin{array}{c}\text { Toujours inacceptable } \\
\text { OR }\end{array}$} & IC (95\%) & \multicolumn{2}{c}{ Toujours acceptable } \\
OR & IC (95\%) \\
Âge & & & & \\
$\quad 18-24$ ans & 1,81 & $0,93-3,50$ & 0,63 & $0,32-1,26$ \\
$25-34$ ans & 0,69 & $0,36-1,33$ & 0,67 & $0,36-1,25$ \\
35-44 ans & 0,94 & $0,52-1,71$ & 0,80 & $0,44-1,45$ \\
$45-54$ ans & 1,15 & $0,62-2,13$ & 1,08 & $0,59-1,98$ \\
$55-64$ ans & 1,56 & $0,83-2,94$ & 0,96 & $0,50-1,86$ \\
65 ans et plus (référence) & - & - & - & -
\end{tabular}

Revenu

\begin{tabular}{|c|c|c|c|c|}
\hline & $0,82 * *$ & $0,71-0,94$ & 1,00 & $0,88-1,14$ \\
\hline \multicolumn{5}{|l|}{ Langue maternelle } \\
\hline Français & 0,69 & $0,39-1,24$ & 1,64 & $0,77-3,51$ \\
\hline Anglais & 1,08 & $0,51-2,27$ & $2,72 *$ & $1,10-6,72$ \\
\hline Autre (référence) & - & - & - & - \\
\hline \multicolumn{5}{|l|}{ Identification religieuse } \\
\hline Catholique & 1,42 & $0,73-2,76$ & 0,72 & $0,43-1,23$ \\
\hline Autres religions & $2,45^{*}$ & $1,09-5,63$ & 0,44 & $0,19-1,02$ \\
\hline Athée/agnostique (référence) & - & - & - & - \\
\hline \multicolumn{5}{|l|}{ Pratique religieuse } \\
\hline & $1,43 * *$ & $1,14-1,78$ & 0,82 & $0,63-1,07$ \\
\hline
\end{tabular}

TABLEAU 3

RÉGRESSION MULTINOMIALE DE L'OPINION SUR L'EUTHANASIE LORSQU'IL Y A ESPOIR DE GUÉRISON ( $\mathrm{N}=935)$

Toujours inacceptable Toujours acceptable

OR IC $(95 \%) \quad$ OR IC $(95 \%)$

$\begin{array}{lcccc}\text { Âge } & & & & \\ 18-24 \text { ans } & 2,05^{*} & 1,08-3,93 & 0,85 & 0,40-1,81 \\ 25-34 \text { ans } & 0,71 & 0,37-1,37 & 0,82 & 0,41-1,63 \\ 35-44 \text { ans } & 0,94 & 0,53-1,67 & 0,68 & 0,35-1,33 \\ 45-54 \text { ans } & 1,11 & 0,61-2,01 & 0,96 & 0,49-1,87 \\ 55-64 \text { ans } & 1,43 & 0,78-2,61 & 0,63 & 0,29-1,37 \\ 65 \text { ans et plus (référence) } & - & - & - & -\end{array}$

Revenu

Langue maternelle

$0,80^{* * *} \quad 0,70-0,92 \quad 0,96 \quad 0,83-1,12$

Français

$0,50 * \quad 0,27-0,91 \quad 0,42 * \quad 0,21-0,83$

Anglais

$1,00 \quad 0,47-2,13 \quad 1,45 \quad 0,63-3,32$

Autre (référence)

Identification religieuse

Catholique

Autres religions

Athée/agnostique (référence)

Pratique religieuse

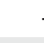$$
-\quad-
$$$$
\text { - } \quad-
$$

$\begin{array}{llll}1,38 & 0,72-2,63 & 0,53 * & 0,29-0,97 \\ 2,59 * & 1,15-5,81 & 0,47 & 0,20-1,14\end{array}$

- $\quad-$$$
-
$$

\begin{tabular}{llllll}
\multicolumn{1}{c}{$1,48 * * *$} & $1,19-1,84$ & 0,92 & $0,68-1,25$ \\
\hline OR: odds ratio & IC: intervalle de confiance & $* \mathrm{p}<0,05$ & $* * \mathrm{p}<0,01$ & $* * * \mathrm{p}<0,001$
\end{tabular}

(2,5 fois) et pratiquent plus leur religion ( 1,4 fois), tandis que les personnes qui considèrent toujours l'euthanasie comme acceptable sont plus nombreuses parmi celles qui ont l'anglais comme langue maternelle [référence=allophone] (2,7 fois).

Dans la situation d'une personne qui demande l'euthanasie lorsqu'il y a espoir de guérison (tableau 3), le modèle retenu explique $16 \%$ de la variance et contient les mêmes variables que dans la situation précédente. Comparativement aux personnes qui ont adopté une opinion plus restrictive dans cette condition, celles qui considèrent toujours l'euthanasie comme inacceptable sont 2 fois plus nombreuses parmi le groupe d'âge 18-24 ans [référence $=65$ ans et plus] et parmi celles qui s'identifient à une religion autre que catholique [référence $=$ athée] (2,6 fois), elles pratiquent plus leur religion ( 1,5 fois) et ont un revenu moins élevé (1,3 fois) ; elles sont également 2 fois moins nombreuses parmi celles dont la langue maternelle est le français [référence $=$ allophone]. Quant aux personnes qui considèrent l'euthanasie comme toujours acceptable même s'il y a espoir de guérison, elles sont 2,4 fois moins nombreuses parmi celles dont la langue maternelle est le français [référence=allophone] et moins nombreuses à s'identifier à la religion catholique [référence $=$ athée] $(1,9$ fois) que celles qui ont changé d'opinion. Les résultats des analyses bivariées révèlent toutefois une différence significative entre les 3 groupes en ce qui concerne le lieu de résidence $(\mathrm{Phi}=0,11 ; \mathrm{p}<0,01)$ : les personnes vivant sur l'île de Montréal sont moins nombreuses à changer d'opinion lorsqu'il y a espoir de guérison $(48,2 \%)$ que celles qui habitent dans les autres régions du Québec (59,5\%).

Lorsqu'il s'agit de l'euthanasie pour cause de souffrance psychologique (tableau 4), les résultats sont quelque peu différents de ceux obtenus lors des analyses bivariées. Seules des distinctions entre le groupe qui considère l'euthanasie comme toujours inacceptable et celui qui change d'opinion demeurent significatives : tout comme pour la situation où il y a espoir de guérison, les personnes qui considèrent l'euthanasie comme toujours inacceptable sont 2 fois plus nombreuses parmi le groupe d'âge $18-24$ ans [référence $=65$ ans et plus] et parmi celles qui s'identifient à une religion autre que catholique [référence $=$ athée] (3,8 fois). Elles pratiquent plus fréquemment leur religion ( 1,6 fois) et ont un revenu moins élevé (1,3 fois). Ce modèle explique $14 \%$ de la variance. Les résultats des analyses bivariées révélaient toutefois une différence significative entre les 3 groupes en ce qui concerne le niveau de scolarité complété $(\mathrm{F}(2,911)=3,40 ; \mathrm{p}<0,05)$ et la langue maternelle $(\mathrm{Phi}=0,14 ; \mathrm{p}<0,01)$. Les personnes qui ont changé d'opinion ont déclaré un niveau de scolarité moyen légèrement plus élevé $(M=1,9$; ÉT $=0,9)$ que celles qui considèrent toujours l'euthanasie comme acceptable dans cette condition $(\mathrm{M}=1,7 ; \mathrm{E} T=0,9)$; celles qui considèrent toujours l'euthanasie comme inacceptable ont déclaré un niveau de scolarité moyen intermédiaire $(\mathrm{M}=1,8 ; \mathrm{E} T=0,9)$. Pour ce qui est de la langue maternelle, la distinction se fait plutôt parmi les groupes ayant 
des opinions tranchées: les francophones sont moins nombreux que les allophones à considérer l'euthanasie comme toujours inacceptable $(24,6 \% / 43,0 \%)$ et plus nombreux à considérer l'euthanasie comme toujours acceptable (22,7\%/12,7\%).

Dans la situation d'une personne très âgée qui demande l'euthanasie (tableau 5), le patron de relation entre les variables est exactement le même que celui de la situation où il y a espoir de guérison et lors de souffrances psychologiques, pour ce qui distingue le groupe «change d'opinion » et ceux qui considèrent l'euthanasie comme "toujours inacceptable », quant à l'âge [OR 2,2; IC 1,1-4,3], le revenu [OR 0,8; IC 0,7-0,9], l'identification à une religion [OR 4,0; IC 1,8-8,8] et la pratique religieuse [OR 1,5; IC 1,2-1,8]. Toutefois, à comparer à ceux qui changent pour une opinion plus restrictive face à l'euthanasie dans le cas d'une personne très âgée, ceux qui considèrent l'euthanasie comme toujours acceptable sont 2 fois moins nombreux parmi les groupes d'âge de 25 à 34 ans et de 35 à 44 ans que parmi le groupe de 65 ans et plus. Les analyses bivariées ont cependant révélé une différence significative entre les groupes sur la variable «langue maternelle» $(\mathrm{Phi}=0,13$; $\mathrm{p}<0,01)$ : les francophones sont moins nombreux que les allophones à considérer l'euthanasie comme toujours inacceptable $(24,2 \% / 43,0 \%)$ mais plus nombreux à changer d'opinion sur l'euthanasie sur la base du critère de l'âge $(51,9 \% / 39,2 \%)$.

\section{DISCUSSION}

Cette étude démontre que les attitudes de la population québécoise sur l'euthanasie sont complexes et différenciées selon les conditions proposées. Ces résultats soutiennent en partie l'idée proposée par Emanuel (2002) selon laquelle la meilleure manière de comprendre les attitudes sur l'euthanasie consiste à observer la « Règle des trois », à savoir que le tiers de la population aurait une opinion favorable à l'euthanasie quelles que soient les conditions, un autre tiers aurait des opinions différenciées selon les conditions de vie et de santé proposées, et un dernier tiers démontrerait une opposition ferme face à l'euthanasie. Dans le cas de cette étude, $16 \%$ des répondants considèrent l'euthanasie comme acceptable même lorsqu'il y a espoir de guérison (condition qui a récolté le moins d'appui), environ $25 \%$ considèrent l'euthanasie comme inacceptable, ce qui implique qu'environ 59\% présentent une opinion différente selon les situations. Cet écart observé avec la «Règle des trois » peut s'expliquer par le caractère plus libéral de la population québécoise puisqu'elle est la province la plus favorable

TABLEAU 4

RÉGRESSION MULTINOMIALE DE L'OPINION SUR L'EUTHANASIE LORS DE SOUFFRANCES PSYCHOLOGIQUES OU MORALES CONTINUELLES (N=914)

\begin{tabular}{|c|c|c|c|c|}
\hline & \multicolumn{2}{|c|}{ Toujours inacceptable } & \multicolumn{2}{|c|}{ Toujours acceptable } \\
\hline & OR & IC $(95 \%)$ & OR & IC $(95 \%)$ \\
\hline \multicolumn{5}{|l|}{ Âge } \\
\hline 18-24 ans & $2,14 *$ & $1,09-4,21$ & 1,28 & $0,63-2,61$ \\
\hline $25-34$ ans & 0,65 & $0,34-1,27$ & 0,73 & $0,38-1,43$ \\
\hline $35-44$ ans & 0,79 & $0,43-1,42$ & 0,53 & $0,28-1,02$ \\
\hline 45-54 ans & 1,06 & $0,57-1,97$ & 1,13 & $0,60-2,14$ \\
\hline $55-64$ ans & 1,43 & $0,76-2,71$ & 1,02 & $0,51-2,04$ \\
\hline 65 ans et plus (référence) & - & - & - & - \\
\hline \multicolumn{5}{|l|}{ Revenu } \\
\hline & $0,78 * *$ & $0,68-0,90$ & 0,89 & $0,77-1,03$ \\
\hline \multicolumn{5}{|l|}{ Identification religieuse } \\
\hline Catholique & 1,63 & $0,85-3,10$ & 0,96 & $0,54-1,69$ \\
\hline Autres religions & $3,76 * *$ & $1,71-8,37$ & 0,70 & $0,29-1,69$ \\
\hline Athée/agnostique (référence) & - & - & - & - \\
\hline \multicolumn{5}{|l|}{ Pratique religieuse } \\
\hline & $1,59 * * *$ & $1,27-1,99$ & 1,10 & $0,84-1,44$ \\
\hline
\end{tabular}

à l'euthanasie lorsque comparée aux autres provinces canadiennes, et ce, depuis 1989 (Gallup Canada, 1989 à 2002). D'autres études seraient toutefois nécessaires afin de mieux comprendre cette proportion importante, chez la population québécoise, d'opinions différenciées selon les conditions de vie et de santé proposées. Peut-être que ceci démontre une grande ouverture des gens au sujet de l'euthanasie dans le contexte d'une personne souffrante en phase terminale, mais une opposition importante à une telle pratique pour toute autre condition que celle-ci.

De façon plus spécifique, cette étude révèle que seulement $16 \%$ des répondants jugent la pratique de l'euthanasie acceptable lorsqu'il y a espoir de guérison comparativement à 69,6\% dans un contexte de maladie terminale. Ainsi, la proximité entre le geste et l'imminence du décès est une condition importante; il semble donc plus acceptable de provoquer la mort à quelques jours ou semaines du décès naturel plutôt que des mois et même des années avant. Cela corrobore l'idée selon laquelle l'euthanasie renvoie dans l'imaginaire collectif à une situation où la personne est en fin de vie. De plus, le fait que seulement $25 \%$ des répondants soient toujours en faveur de l'euthanasie lorsque l'élément de la souffrance est absent appuie également l'idée selon laquelle la pratique de l'euthanasie est conditionnée par la souffrance de la personne. Il serait également intéressant de vérifier si ces conditions inhérentes à la conception même de l'euthanasie perdurent dans le temps ou si, au contraire, ces notions perdent de leur importance.
Dans le cas d'une personne qui n'est pas atteinte d'une maladie physique mais qui a des souffrances psychologiques ou morales continuelles, c'est environ une personne sur cinq qui considère l'euthanasie comme acceptable. Cette donnée est intéressante puisqu'elle révèle que peu de gens au Québec sont prêts à envisager l'euthanasie dans une telle situation. La souffrance psychologique est toutefois reconnue comme un critère acceptable au point de vue légal aux PaysBas; ce qui avait soulevé la polémique et divisé les partisans de la cause de l'euthanasie au moment des pourparlers concernant l'adhésion à cette condition (Sénat du Canada, 1995). D'une situation où la personne devait être en phase terminale et en proie à des souffrances intolérables pour avoir accès à l'euthanasie, la souffrance psychologique intense est devenue une raison acceptable pour revendiquer un droit à la mort (Legemaate et al., 1997). Pour l'Association médicale royale néerlandaise, la justification d'un tel changement était celle de n'exercer aucune discrimination envers la souffrance psychologique puisque c'est l'expérience de la souffrance et son intensité, plutôt que sa nature (physique ou psychologique) et son origine (maladie physique ou mentale), qui importent (Gevers et al., 1998). Le suivi de l'évolution de l'opinion sur l'euthanasie dans le cas de la souffrance mentale est primordial et pourrait, à notre avis, permettre d'infirmer ou de confirmer l'argument de la pente glissante selon lequel une ouverture à l'égard de l'euthanasie, aussi minime soit-elle, engendrerait une acceptation croissante des raisons et situations pour lesquelles l'euthanasie est jugée acceptable. 


\section{TABLEAU 5}

RÉGRESSION MULTINOMIALE DE L'OPINION SUR L'EUTHANASIE LORSQUE LA PERSONNE EST TRÈS ÂGÉE $(N=928)$

Groupe de référence:
Change d'opinion
Toujours inacceptable

OR

IC $(95 \%)$

Toujours acceptable

OR IC (95\%)

Âge

18-24 ans

$2,20 *$

$1,11-4,34$

1,05

$0,54-2,61$

25-34 ans

0,62

$0,32-1,19$

0,48 *

0,80

$0,44-1,46$

$0,51^{*}$

$0,26-0,91$

35-44 ans

0,92

$0,50-1,71$

0,61

$0,28-0,92$

1,44

$0,76-2,72$

0,77

$0,33-1,12$

55-64 ans

65 ans et plus (référence)

$-$

$-$

Revenu

Identification religieuse

Catholique

Autres religions

Athée/agnostique (référence)

Pratique religieuse

$0,69-0,91$

0,91

$0,80-1,05$

$$
0,79 * *
$$

1,67

0,87-3,19

1,05

1,02

$0,61-1,82$

$4,00 * *$

$1,82-8,83$

$-$

-

$1,48^{* * *} \quad 1,18-1,84 \quad 0,91 \quad 0,70-1,18$

OR: odds ratio IC: intervalle de confiance

* $\mathrm{p}<0,05$

$* * \mathrm{p}<0,01$

Le même constat pourrait être fait dans le cas d'une personne très âgée mais qui n'est pas atteinte d'une maladie terminale. De fait, une proportion semblable d'environ une personne sur cinq considère l'euthanasie comme acceptable dans une telle condition. Avec le vieillissement de la population et la rareté croissante des ressources dans le domaine de la santé, il serait intéressant de voir l'évolution de la pensée des Québécoises et Québécois sur cette question. Déjà, des remarques ont été faites dans l'actualité quant à d'éventuels problèmes dans le réseau des soins de santé en raison du vieillissement de la population et des coûts sociaux qui y sont associés (KellyGagnon, 2003), et certains avancent même qu'il serait bénéfique de légaliser l'euthanasie en raison des économies que cela pourrait générer (Scott, 2001). La rationalisation des soins de santé sur la base du critère de l'âge est un débat déjà bien entamé, surtout aux États-Unis, bien que cette idée récolte peu d'adeptes (Kapp, 2001 ; Zweibel, Cassel et Karrison, 1993).

À notre connaissance, il s'agit de la seule étude qui se soit intéressée aux caractéristiques mêmes des personnes qui ont des opinions divergentes selon diverses conditions. Les résultats révèlent que les distinctions se font plutôt sentir lorsque ce groupe est comparé à celui des personnes qui détiennent des opinions défavorables sur l'euthanasie de manière générale. Ces distinctions sont, à quelques exceptions près, les mêmes que celles qui différencient les personnes ayant des attitudes favorables sur l'euthanasie de celles ayant des attitudes défavorables; un revenu ou un statut socioéconomique plus faible (Decesare,
2000; Gilman et al., 1997; Suarez-Almazor et al., 1997), l'appartenance à une religion et une pratique plus fréquente de celle-ci (Decesare, 2000; Genuis et al., 1994; Gilman et al., 1997 ; Pollard, 2001 ; SuarezAlmazor et al., 1997; van der Maas et al., 1995) caractérisent plus fréquemment les personnes ayant des attitudes défavorables à l'égard de l'euthanasie. Un résultat étonnant de cette étude concerne l'âge, puisque les jeunes âgés de 18 à 24 ans sont plus nombreux que les personnes de 65 ans et plus à considérer l'euthanasie comme inacceptable et à ne pas changer d'opinion selon les conditions de vie et de santé proposées. Bien qu'il soit difficile d'interpréter une telle donnée, cela pourrait traduire un plus grand conservatisme des jeunes en raison du peu de réflexion et/ou d'expériences personnelles jusqu'à maintenant en lien avec la maladie et la mort. Il serait intéressant d'investiguer cet aspect dans le cadre d'une prochaine étude.

Comme à la base les personnes qui changent d'opinion selon les situations détiennent une opinion favorable à l'euthanasie dans un contexte de maladie incurable et de souffrance, il est particulièrement intéressant de savoir ce qui les différencie de celles qui sont favorables à l'euthanasie peu importe les conditions de vie et de santé que nous avons proposées. Lorsque les éléments de base auxquels l'euthanasie est généralement associée sont absents, c'est-à-dire dans les contextes où il n'y a pas de souffrance ou lorsqu'il y a espoir de guérison, la langue maternelle et l'identification religieuse semblent jouer un rôle. Dans la situation où il y a espoir de guérison, les personnes dont la langue maternelle est le français, qui sont également plus nombreuses parmi celles qui s'identifient à la religion catholique, sont plus portées à changer d'idée et à considérer l'euthanasie comme inacceptable dans cette condition comparativement aux personnes qui se disent athées, qui sont plus enclines à favoriser l'euthanasie peu importe les conditions. Le même parallèle pourrait être fait avec la situation où il n'y a pas de souffrance pour ce qui concerne le lien entre la langue maternelle, le lieu de résidence et la religion dans le contexte québécois. Pour ce qui est de la situation où la personne a des souffrances psychologiques ou morales continuelles, les résultats des analyses bivariées ont révélé que les personnes ayant un niveau de scolarité plus élevé sont plus nombreuses à changer d'idée et à considérer alors que l'euthanasie est inacceptable. Dans l'analyse multivariée, la variance associée à l'éducation a probablement été absorbée par le revenu; ces deux variables étant toutes deux des indicateurs de statut socioéconomique. Malgré cela, un tel résultat pourrait indiquer que les gens plus éduqués pensent que la souffrance psychologique n'est pas immuable et qu'elle peut être soulagée et traitée par des soins appropriés. De plus, il est intéressant de voir que les personnes âgées de plus de 65 ans, à comparer à celles qui ont entre 25 et 44 ans, sont plus nombreuses à considérer l'euthanasie comme toujours acceptable pour la situation mettant en cause une personne âgée, peut-être en raison de leur proximité avec la situation hypothétique. Il serait intéressant de creuser un peu plus cette question et de voir si une telle possibilité relèverait plutôt pour eux d'un droit de choisir ou d'un devoir. De telles hypothèses devraient être examinées dans le futur.

Cette étude comporte certaines limites. Malgré un taux de réponse acceptable pour un sondage d'opinion publique $(50 \%)$, il est possible que les non-répondants puissent constituer un groupe différent des répondants à l'étude, bien que ce problème soit partiellement contourné par la procédure de pondération qui démontre que les répondants ne se différencient pas vraiment de la population québécoise sur un ensemble de caractéristiques sociodémographiques. Les conditions de vie et de santé étudiées et présentées dans cet article ne sont pas exhaustives, elles ont toutefois été choisies sur la base des débats actuels sur la question de l'euthanasie. De plus, d'autres prédicteurs du changement d'opinion selon les conditions de vie et de santé devraient être étudiés tels que des facteurs d'ordre culturel, tout comme des expériences personnelles avec la maladie et le processus du mourir, puisque nos modèles n'expliquaient qu'entre $14 \%$ et 
$16 \%$ de la variance des scores de la variable dépendante.

En conclusion, les résultats de cette étude apportent des informations importantes susceptibles d'éclairer des discussions et débats futurs autour de la question de l'euthanasie au Québec ou au Canada. Cette étude révèle que la population québécoise est majoritairement favorable à l'euthanasie dans un contexte de maladie incurable et de souffrance, mais qu'elle est peu encline à considérer une telle pratique comme acceptable dans d'autres conditions, entre autres lorsque la personne ne souffre pas, lorsqu'il y a espoir de guérison, lorsque la personne a des souffrances psychologiques ou morales continuelles, ou lorsqu'il s'agit seulement du fait que la personne est très âgée. De tels résultats montrent que le débat sur l'euthanasie n'est pas simple; ils nous renseignent sur les volontés du public en matière d'euthanasie, mais nous éclairent également sur les nuances à apporter aux résultats des sondages sur la question. De plus, cette étude aide à mieux comprendre ce qui semble influencer un changement d'opinion, ce qui n'avait pas encore été étudié auparavant. Les résultats fournissent également des données de base en regard d'un suivi de l'évolution des mentalités sur l'euthanasie et sur différentes conditions dans lesquelles elle est considérée comme acceptable ou non. L'étude des changements d'opinion à travers le temps selon certaines conditions pourrait ainsi permettre d'infirmer ou de corroborer l'argument de la pente glissante. Bref, le suivi de l'opinion de la population québécoise et canadienne sur ces questions semble primordial afin d'alimenter un débat adapté à notre contexte social, culturel et politique.

\section{Bibliographie}

ACHILLE, M.A. et J.R.P. OGLOFF (1997). "When is a request for assisted suicide legitimate? Factors influencing public attitudes toward euthanasia», Canadian Journal of Behavioural Science, vol. 29, $\mathrm{n}^{\circ}$ 1, p. 19-27.

DECESARE, M.A. (2000). «Public attitudes toward euthanasia and suicide for terminally ill persons : 1977 and 1996 », Social Biology, vol. 47, no 3-4, p. 264-276.

EMANUEL, E.J. (2002). "Euthanasia and physician-assisted suicide: A review of the empirical data from the United States", Archives of Internal Medicine, vol. 162, $\mathrm{n}^{\circ} 2$, p. $142-152$.

EMANUEL, E.J., E.R. DANIELS, D.L. FAIRCLOUGH et B.R. CLARRIDGE (1998). "The practice of euthanasia and physicianassisted suicide in the United States: Adherence to proposed safeguards and effects on physicians ", Journal of the American Medical Association, vol. 280, n 6, p. 507-513.

GALLUP CANADA (1968-). THE GALLUP REPORT, Toronto, Canadian Institute of Public Opinion.
GENUIS, S.J., S.K. GENUIS et W.-C. CHANG (1994). «Public attitudes toward the right to die ", Canadian Medical Association Journal, vol. 150, n 5 , p. 701-708.

GEVERS, S. et J. LEGEMAATE (1998). "Physician assisted suicide in psychiatry: An analysis of case law and professional opinions », dans D.C. THOMASMA T. KIMBROUGH-KUSHNER, G.K. KIMSMA et C. CIESIELSKI-CARLUCCI (dir.), Asking to Die: Inside the Dutch Debate about Euthanasia, Dordrecht, Kluwer Academic Publishers, p. 71-91.

GILMAN, P.A., L.L. MERRILL et J.L. REID (1997). "Attitudes toward euthanasia », Perceptual and Motor Skills, vol. 84, n 1, p. 317318.

KAPP, M.B. (2001). «Economic influences on end-of-life care: Empirical evidence and ethical speculation », Death Studies, vol. 25, $\mathrm{n}^{\circ}$ 3, p. 251-263.

KELLY-GAGNON, M. (2003). «Le vieillissement et le coût de la santé », La Presse, samedi 15 février, p. 12

LAVRAKAS, P.J. (1987). Telephone Survey Methods: Sampling, Selection, and Supervision, Newbury Park, Californie, Sage.

LEGEMAATE, J. et J.K.M. GEVERS (1997). "Physician-assisted suicide in psychiatry: Developments in the Netherlands ", Cambridge Quarterly of Healthcare Ethics, vol. 6, $\mathrm{n}^{\circ} 2$, p. $175-188$.

MCINERNEY, F. et C. SEIBOLD (1995). "Nurses' definitions of attitudes towards euthanasia », Journal of Advanced Nursing, vol. $22, \mathrm{n}^{\circ} 1$, p. 171-182.

MACDONALD, W.L. (1998). "Situational factors and attitudes toward voluntary euthanasia », Social Science and Medicine, vol. 46, $\mathrm{n}^{\mathrm{o}} 1$, p. $73-81$

MARCOUX, I., B.L. MISHARA et C. DURAND (2007). "Confusion between euthanasia and other end-of-life decisions: Influences on public opinion poll results », Canadian Journal of Public Health, vol. 98, $\mathrm{n}^{\circ} 3$, p. 235-239.

MULLENS, A. (1994). Leadership moribond en matière d'euthanasie, ColombieBritannique, Bourse Atkinson.

O'NEILL C., D. FEENAN, C. HUGHES et D.A. MCALISTER (2003). "Physician and family assisted suicide: Results from a study of public attitudes in Britain », Social Science and Medicine, vol. 57, $\mathrm{n}^{\circ}$ 4, p. 721-731.

O'NEILL, C.O., D. FEENAN, C. HUGUES, D.A. MCALISTER (2002). «Attitudes to physician assisted suicide: Results from a study of public attitudes in Britain », Journal of Medical Ethics, vol. 28, n 1 , p. 52.

POLLARD, J. (2001). The Impact of Religious Affiliation and Religious Practices on Attitudes Toward Euthanasia, mémoire de maîtrise, Toronto, York University.

ROY MORGAN RESEARCH CENTRE (1996). Morgan Polls on Medically Assisted Death, Finding $N^{o}$ 2933, Melbourne Roy Morgan Research Centre.

SCOTT, N. J. (2001). «Bonne mort, en d'autres termes », La Presse, lundi 28 mai, p. 12.
SÉNAT DU CANADA (1995). De la vie et de la mort, publication gouvernementale du Canada.

SILVEIRA, M.J., A. DIPIERO, M.S. GERRITY et C. FEUDTNER (2000). "Patients' knowledge of options at the end of life: Ignorance in the face of death », Journal of the American Medical Association, vol. 284, $\mathrm{n}^{\circ} 19$, p. 2483-2488.

SINGER, P.A., S. CHOUDHRY, J. ARMSTRONG, E.M. MESLIN et F.H. LOWY (1995). "Public opinion regarding end-oflife decisions: Influence of prognosis, practice and process ", Social Science and Medicine, vol. 41, $\mathrm{n}^{\circ}$ 11, p. 1517-1521.

SUAREZ-ALMAZOR, M.E., M. BELZILE et E. BRUERA (1997). "Euthanasia and physician-assisted suicide: A comparative study of physicians, terminally ill cancer patients, and the general population ", Journal of Clinical Oncology, vol. 15, $\mathrm{n}^{\circ}$ 2, p. 418-427.

VAN DER MAAS, P.J., L. PIJNENBORG, J.M. VAN DELDEN (1995). "Changes in Dutch opinions on active euthanasia, 1996 through 1991 », Journal of the American Medical Association, vol. 273, $\mathrm{n}^{\circ}$ 18, p. 1411-1414.

WADE, C.H. et M.D. ANGLIN (1987). «Factors influencing decisions to terminate life », Social Biology, vol. 34, $\mathrm{n}^{\circ}$ 1-2, p. 37-46.

WILLIAMS, N., C. DUNFORD, A. KNOWLES et J. WARNER (2007). " Public attitudes to life-sustaining treatments and euthanasia in dementia », International Journal of Geriatric Psychiatry, vol. 22, $\mathrm{n}^{\circ} 12$, p. 1229-1234, disponible en ligne, <www3.interscience. wiley.com/cgi-bin/fulltext/114250510/ PDFSTART>.

ZWEIBEL, N.R., C.K. CASSEL, T. KARRISON (1993). "Public attitudes about the use of chronological age as a criterion for allocating health care resources ", The Gerontologist, vol. $33, \mathrm{n}^{\circ} 1$, p. 74-80.

\section{Notes}

1. Au moment de l'étude, l'auteure avait obtenu une bourse doctorale du Fonds québécois de la recherche sur la société et la culture (FQRSC) et la collecte de données a été réalisée grâce à une subvention octroyée par le Centre de recherche sur le suicide et l'euthanasie (UQAM).

2. Les situations présentées étant plus restrictives que la question générale sur l'euthanasie, il n'était pas approprié de questionner les personnes qui avaient déjà répondu par la négative à la question générale.

3. Les ménages collectifs (par ex.: résidences pour personnes âgées, couvents, prisons) ne sont pas représentés dans l'échantillon.

4. À part le lieu de résidence de Québec métropolitain, où le nombre de répondants s'élève à $20 \%$ dans l'échantillon au lieu de $10 \%$ dans la population.

5. Les résultats obtenus sont sensiblement les mêmes qu'avec la méthode standard ou hiérarchique. La méthode backward stepwise a donc été retenue puisqu'elle permet une optimisation du modèle d'un point de vue statistique. 